\title{
La « lucumisation » des cultes d'origine africaine à Cuba : le cas de Sagua la Grande
}

Silvina Testa

\section{OpenEdition}

Journals

Édition électronique

URL : https://journals.openedition.org/jsa/2853

DOI : 10.4000/jsa.2853

ISSN : 1957-7842

Éditeur

Société des américanistes

Édition imprimée

Date de publication : 5 janvier 2005

Pagination : 113-138

ISSN : 0037-9174

\section{Référence électronique}

Silvina Testa, «La « lucumisation » des cultes d'origine africaine à Cuba : le cas de Sagua la

Grande ", Journal de la Société des américanistes [En ligne], 91-1 | 2005, mis en ligne le 10 janvier 2010, consulté le 03 septembre 2022. URL : http://journals.openedition.org/jsa/2853 ; DOI : https://doi.org/ $10.4000 /$ jsa. 2853 


\title{
LA « LUCUMISATION » \\ DES CULTES D'ORIGINE AFRICAINE À CUBA : \\ LE CAS DE SAGUA LA GRANDE
}

\author{
Silvina TESTA *
}

\begin{abstract}
La santería ou « religion lucumí » est née dans la région occidentale de Cuba, notamment dans les villes de La Havane et Matanzas. Dans les années suivant l'abolition de l'esclavage (1886), cette religion a connu un processus de diffusion vers l'intérieur du pays créant ainsi une véritable « lucumisation » des cultes d'origine africaine. Dans cet article on analyse son expansion vers l'est de l'île, à travers le cas de la ville de Sagua la Grande. [Mots clés : cabildos, sociétés afro-cubaines, religions afro-cubaines, santería, lucumisation, Cuba, Sagua la Grande.]
\end{abstract}

La « lucumización » de los cultos de origen africano en Cuba : el caso de Sagua la Grande. La santería o " religión lucumi » nació en la región occidental de Cuba, principalmente en las ciudades de La Habana y Matanzas. En los años posteriores a la abolición de la esclavitud (1886), esta religión se expandió hacia otras regiones del país creando un verdadero proceso de « lucumización » de los cultos de origen africano. En este artículo se analiza la expansión de este proceso hacia el este de la isla, a través del caso de la ciudad de Sagua la Grande. [Palabras claves : cabildos, sociedades afrocubanas, religiones afrocubanas, santería, lucumización, Cuba, Sagua la Grande.]

The "lukumisation" of the religions of African origin in Cuba : the case of Sagua la Grande. Santeria or the "Lukumi religion " was born in the western part of Cuba, especially in Havana and Matanzas. After the abolition of slavery (1886) this religion spread to other parts of the island, and in so doing created a process of «lukumisation " of the other religions of African origin. This article will analyse the spread of Santeria towards the eastern part of Cuba and look specifically at the case study of Sagua La Grande. [Key words : cabildos, Afro-Cuban societies, Afro-Cuban religions, santeria, lukumisation, Cuba, Sagua la Grande.]

* Laboratoire d'ethnologie et de sociologie comparative, UMR 7535, Université de Paris XNanterre [silvina_testa@hotmail.com].

Journal de la Société des Américanistes, 2005, 91-1, pp. 113-138. O Société des Américanistes. 
Pour une large majorité des pratiquants havanais des religions d'origine africaine, le seul nom de Sagua la Grande renvoie aussitôt aux « racines » (la mata) de leurs cultes. C'est l'idée de la "campagne » ${ }^{1}$, par opposition à la « ville » et, par conséquent, l'idée d'une certaine " pureté » qui différerait des pratiques de la capitale, qui est mise en avant. Mais c'est aussi la représentation de Sagua comme point d'origine des cultes contemporains, comme si, dans cette ville de l'intérieur du pays, on pouvait trouver certaines marques fondatrices des pratiques de la capitale. Or l'histoire démontre le processus inverse : Sagua la Grande est une ville héritière des religions d'origine afro-cubaine nées à l'ouest ${ }^{2}$.

Même si, aujourd'hui, la santería est considérée comme une religion cubaine parce qu'elle est pratiquée d'un extrême à l'autre de l'île, à l'origine elle était exclusivement occidentale. En 1942, Rómulo Lachatañeré (2001, p. 263) affirmait que le culte « lucumí » se manifestait de manière intense à La Havane et dans certaines villes de la province de Matanzas. Mais, bien avant cela, les transformations survenues à Cuba entre la fin du XIX ${ }^{\mathrm{e}}$ et le début du $\mathrm{xx}^{\mathrm{e}}$ siècle avaient déjà créé le terrain propice à l'« exportation » de ces pratiques vers d'autres régions du pays, notamment le centre de l'île (Figure 1).

Le cas de Sagua la Grande est exemplaire à plus d'un titre. En nous appuyant sur les institutions qui ont servi de plate-forme à la mobilité des pratiquants entre les régions occidentale et centrale ${ }^{3}$, nous avons retracé le puissant processus d'expansion progressive du culte lucumí et nous avons choisi de l'appeler « lucumisation ". L'analyse de cette dynamique met en évidence la réorganisation profonde qui s'opère dans la religiosité d'origine africaine. Dans cet article, nous tenterons de comprendre comment on est passé des pratiques religieuses familiales à des pratiques individuelles, tandis qu'une organisation jusque-là fondée sur le lignage biologique s'appuie désormais sur le lignage rituel.

\section{LES INSTITUTIONS AFRO-CUBAINES ET LA RELIGION LUCUMI}

Les religions d'origine africaine pratiquées de nos jours à Cuba sont nées pendant la période coloniale et étaient étroitement en relation avec la vie institutionnelle des Africains et de leurs descendants. En effet, les cabildos de nación, d'abord, et les "sociétés d'instruction, secours et entraide" (sociedades de instrucción, socorro y ayuda mutua) ${ }^{4}$, ensuite, sont les lieux où ces religions ont pris forme à La Havane et Matanzas, se sont développées et, postérieurement, ont été « exportées » vers différentes villes du pays.

Les cabildos étaient des institutions réunissant les Africains par nation. La définition la plus ancienne, et néanmoins valable, est celle d'Esteban Pichardo (1985, p. 114). On appelle cabildo « la réunion de Noirs et Noires bossales dans des maisons destinées à cet usage les jours de fêtes, où ils jouent de leurs timbales et tambours et d'autres instruments nationaux, ils chantent et dansent 


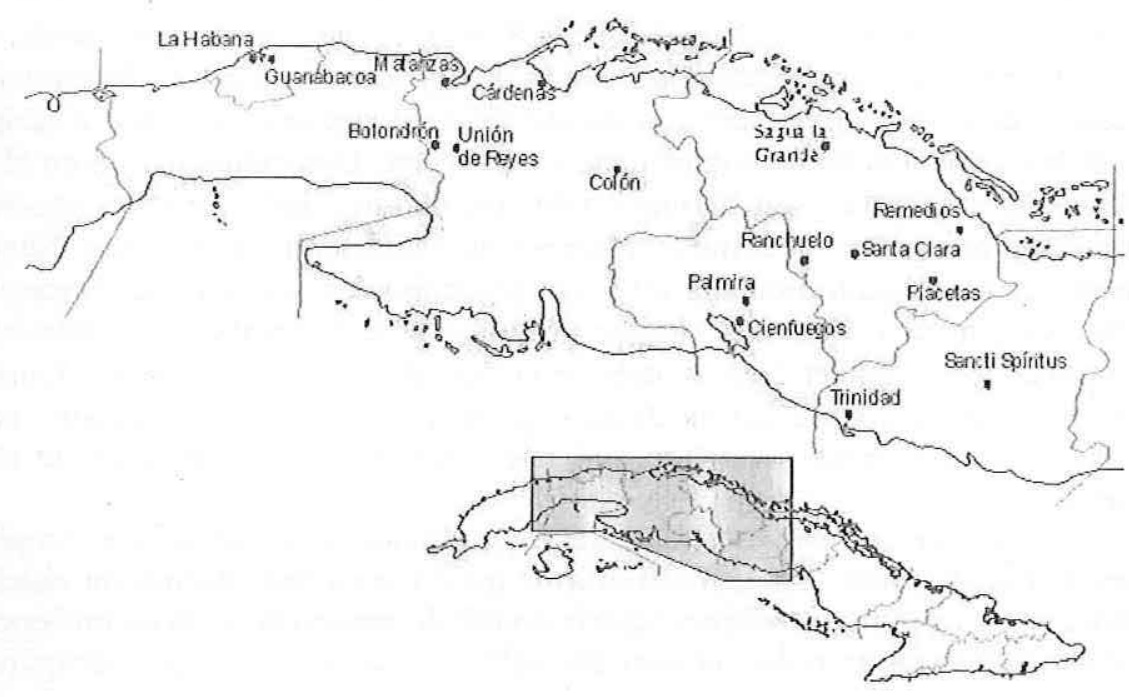

FIG. 1 - Carte de Cuba.

en désordre, avec un bruit d'enfer et constant, sans intermittence. Ils réunissent des fonds et forment une espèce de société de pur divertissement et secours, avec leur caja ${ }^{5}$, leurs contremaître, majordome, roi, reine (sans juridiction). Chaque nation a son cabildo et, de ce fait, on les dénomme cabildo arará, cabildo carabali, etc. ». Ce type d'institution arrive à Cuba par le biais des Espagnols, ce qui fait dire à Fernando Ortiz (1993, p. 58) qu'il s'agit d'une « survivance outremer d'une organisation moyenâgeuse andalouse ", car il existait déjà à Séville des cabildos et des confréries de Gitans, Noirs et mulâtres.

La Fête des Rois mages, célébrée le 6 janvier, était le moment de l'année le plus attendu dans la vie des cabildos : les Africains sortaient dans la rue avec leurs tambours et défilaient habillés à la mode de leur nation d'origine. Mais les cabildos n'étaient pas seulement des institutions récréatives et d'assistance mutuelle : ils avaient aussi des implications politiques, certains ayant joué un rôle considérable dans les conspirations abolitionnistes des Noirs (Deschamps Chapeaux 1971).

À leurs débuts, les cabildos étaient exclusivement destinés aux esclaves africains. Plus tard, ils ont admis les Africains libres et, finalement, les créoles ou " Africains » de la deuxième génération ${ }^{6}$ (Montejo Arrechea 1993). Leur période d'apogée dure approximativement jusqu'en 1860. À partir de là, les cabildos entament leur déclin. Ils cessent d'admettre les créoles à partir de 1868. En 1876, l'Espagne promeut leur transformation en prenant pour modèle les sociétés 
ibériques blanches (loi des associations de 1878). Six ans plus tard, en janvier 1884, on proscrit la sortie de la Fête des Rois et, en avril de la même année, le gouvernement colonial interdit la création de nouveaux cabildos. L'abolition de l'esclavage, en 1886, intervient au moment où le paysage associatif des Africains et de leurs descendants est complètement transformé. Désormais, il n'y aura plus de cabildos : certains sont fermés tandis que d'autres deviennent des sociétés d'instruction, secours et entraide, placées sous l'égide d'un saint patron. Néanmoins, cette réorganisation, qui se voulait généralisable à tout le pays, n'a pas été effective dans toutes les villes, comme à Matanzas, où de nombreux cabildos ont continué à fonctionner bien au-delà de la date de proscription (Hevia Lanier 2002). En effet, il n'y a pas eu de passage obligé du cabildo à la société ; par ailleurs, de nombreuses sociétés sont nées sans avoir préalablement été des cabildos.

Les relations entre les cabildos et l'Église catholique ont toujours été complexes. Fernando Ortiz (1993, p. 60) affirme qu'il y a eu substitution du fétiche africain par un saint catholique et que la double dimension de cabildo-confrérie a permis l'incorporation des cabildos aux églises. Pourtant, on ne peut simplifier ainsi ces rapports car de nombreux cabildos n'ont pas eu de relations avec l'Église catholique, ce qui est le cas à Sagua la Grande.

Nous avons dit que les cabildos et sociedades ont été le point de départ de la création des religions d'origine africaine à Cuba. En effet, c'est au sein de ces institutions que naît ce qui est connu au $\mathrm{xx}^{\mathrm{e}}$ siècle sous le nom de "religion lucumí ». Le vocable " lucumí » est employé à Cuba depuis le Xvi ${ }^{\mathrm{e}}$ siècle, mais son utilisation s'est diversifiée selon l'époque, le contexte et l'auteur. Au fil du temps, ce terme a désigné d'abord un groupe d'esclaves, ensuite leur langue et finalement une religion. Selon Fernando Ortiz (1906), le mot « lucumí » serait une déformation du nom de la région et/ou du royaume Ulcami ou Ulcumi, qui ne serait autre que celui d'Oyo, qui aurait été fondé au $x v^{\mathrm{e}}$ siècle par Oranyan, fils d'Oduduwa, sur les terres de l'actuel Nigeria. Par extension, à leur arrivée à Cuba, étaient appelés « lucumí » tous les esclaves originaires de l'Afrique de l'Ouest, dont les plus nombreux étaient yoruba. À partir de la désignation d'un groupe humain, le sens du vocable « lucumí » a été élargi pour nommer leur langue. Puis, au cours $\mathrm{du} \mathrm{xx}^{\mathrm{e}}$ siècle, il fait surtout référence à un type de pratiques religieuses. Nicolás Angarica ${ }^{7}$ (1990), santero $^{8}$ de grande renommée, va même écrire un texte, " $\mathrm{El}$ lucumí al alcance de todos ", qui s'adresse aux pratiquants et aux chercheurs et qui se veut le modèle de la religion lucumí. L'ouvrage offre un large éventail de rituels, cérémonies, prières et, même, un dictionnaire lucumí-espagnol avec la terminologie utilisée dans la liturgie. En 1942, Rómulo Lachatañeré développera l'idée de " panthéon lucumí », comme un ensemble de divinités (set de deidades), qui n'est pas uniforme et que chaque maison de culte utilise selon son expérience et ses compétences, en donnant la priorité aux divinités qui ont démontré une plus grande efficacité dans la résolution des problèmes des pratiquants. L'ensemble 
des divinités énoncées par Lachatañeré (2001, pp. 100-102) ne sont autres que les orichas ${ }^{9}$ des Yoruba ${ }^{10}$ : Olofín, Olordumare, Odudúa, Obatalá, Baba-lu-Ayé, Orúmbila, Olokún, Yemayá, Ogún, Agayú, Oyá, Changó, Eleguá, Ochún, etc. Cette « religion lucumí » sera aussi appelée « Regla de Ocha » ou " santería », et elle sortira de son berceau occidental pour se répandre dans d'autres régions, notamment le centre du pays.

\section{Aux origines de la religiosité afro-cubaine à Sagua la Grande}

La ville de Sagua la Grande s'est intégrée tardivement à l'économie nationale. Au XIX ${ }^{\mathrm{e}}$ siècle, grâce à la demande internationale de plus en plus forte en sucre, de nouvelles zones de plantations ont été développées à Cuba, en particulier Sagua la Grande ${ }^{11}$. L'essor de la ville est le fruit du déplacement des capitaux occidentaux, notamment de La Havane et de Matanzas, vers le centre du pays, à la recherche de nouvelles terres cultivables (Moreno Fraginals 1978).

Témoin de ce rapide développement, la juridiction de Sagua compte en 1827 2 raffineries de sucre, 59 en 1846, 125 en 1862 (Venegas Delgado 1989). En 1860, Sagua occupe la quatrième place dans la production nationale ${ }^{12}$, produisant $11 \%$ du sucre de l'ensemble de l'île (Rebello 1860). L'accroissement économique local va de pair avec l'introduction massive d'une main-d'œuvre esclave, maind'œuvre sur laquelle a reposé la croissance sucrière cubaine. La présence d'Africains dans la province de Las Villas ${ }^{13}$ est supérieure à la moyenne coloniale du nombre d'esclaves par propriétaire (1/7,9 pour une moyenne de $1 / 7,5)$, ce qui fait de cette province un " centre fondamental de l'expansion esclavagiste du milieu du XIX ${ }^{\mathrm{e}}$ siècle » (ibid., p. 72). De plus, Sagua est devenue l'une des trois villes, avec La Havane et Matanzas, à partir desquelles s'est développée la location d'esclaves. Ainsi, malgré son expansion économique fort tardive, Sagua la Grande a rapidement occupé une place privilégiée à côté des villes phares de l'économie négrière nationale.

Une des modalités cultuelles d'origine africaine, sans doute la plus ancienne, date de cette période. En effet, le $\mathrm{XIX}^{\mathrm{e}}$ siècle est aussi le moment où les premières formes de religiosité apparaissent à Sagua. De cette époque subsistent jusqu'à nos jours quelques pièces rituelles appelées « reliques de famille » (reliquias de familia). Cette dernière expression peut prendre deux sens : le premier renvoie à un corps ou un fragment de corps (restes, ossements) d'un héros, d'un saint, d'un bienheureux, ou à des objets qui lui ont appartenu, qui prennent donc un caractère sacré et à qui les fidèles rendent un culte. Le second est associé à un objet auquel on attache moralement la plus grande valeur; en tant que vestige ou témoin d'un passé valorisé. De ces définitions, nous retiendrons le caractère sacré de l'objet de culte et, principalement, la notion d'un temps révolu. Les reliques de famille que nous trouvons à Sagua sont des pièces rituelles du type funda- 
mento ${ }^{14}$, qui ont été fabriquées et rendues actives par un Africain d'origine ou un créole. D’une manière générale, toutes les pièces rituelles peuvent « rester » ou « partir » ${ }^{15}$ à la mort de leur propriétaire; dans les cas des reliques de famille, elles ne partent jamais. Elles ont une durée qui dépasse le temps de vie d'une personne (de son propriétaire ou de l'héritier) : elles restent systématiquement dans la famille comme des objets d'adoration et de protection. Leur détenteur les lègue avant sa mort à un autre membre de la maisonnée. Aujourd'hui ces reliques sont à la charge d'un descendant de troisième ou quatrième génération de l'Africain fondateur. Malgré le fait qu'elles ne « partent » jamais, les reliques ne se reproduisent pas, c'est-à-dire qu'elles n'« accouchent » pas de nouvelles pièces rituelles ${ }^{16}$. De la même manière, les reliques ne " travaillent » plus, sauf en cas de besoin extrême, comme dans une maladie grave ou une épidémie. Dans ces cas, elles font l'objet de diverses activités rituelles. Cette modalité du culte est directement héritée des esclaves installés dans la région. L'Africain d'origine ou le créole qui a construit et rendu active la relique a créé, avec la pièce rituelle, une descendance religieuse qui va suivre les liens de la parenté biologique. Ainsi, l'assembleur devient nécessairement l'ancêtre de ce nouveau lignage religieux.

Parmi les reliques de famille qui existent encore à Sagua, une est exemplaire. Il s'agit de Changó Fama. Selon ses descendants, cette relique est née au XIX ${ }^{\mathrm{e}}$ siècle dans le quartier San Juan (où elle est toujours), dans une maison légendaire, la casa rumba'a. Changó Fama est créée grâce à Florentina Alfonso, femme africaine lucumí, «fille » (omó) de Changó. Avant de mourir, celle-ci lègue la relique à son fils Margarito Alderete, «fils » d'Obatalá, qui s'en est occupé jusqu'à sa mort. Il la transmet alors à sa nièce Florencia Alfonso, "fille » de Changó. D’après les récits de famille, Florentina, la grand-mère, et Florencia, la petite fille, étaient, toutes deux, l'incarnation de leur oricha tutélaire : quand il y avait une tempête, il fallait sortir dehors et jeter un seau d'eau car, sinon, elles étaient aussitôt possédées par Changó, oricha de la foudre. Florencia a épousé Pedro Arango avec qui elle a eu treize enfants parmi lesquels une des filles, bien évidemment, fut l'héritière de Changó Fama : Emilia Arango Alfonso ${ }^{17}$, également « fille » de Changó (Figure 2).

La casa rumba'a n'existe plus. Faite de bois et de paille, elle a été détruite à la suite d'intempéries. Depuis, la famille Arango-Alfonso en a construit une autre, dans le même quartier, cette fois en bois et tuiles. Cependant, au fur et à mesure que les enfants ont grandi, ils ont quitté le foyer parental et aujourd'hui ils ont entre 55 et 80 ans. De plus, la maison ne pouvait contenir toute la descendance qui compte plus d'une centaine de personnes, toutes générations confondues. Aujourd'hui, la maison qui a remplacé la casa rumba'a n'est plus habitée, elle ne sert qu'à abriter la relique Changó Fama. Elle est devenue le lieu de réunion quotidien de plusieurs des membres habitant à proximité. Cette maison était jusqu'en 1999 en mauvais état. Depuis, toute la famille a apporté sa contribution pour refaire les murs en briques. L'engagement de tous vis-à-vis de Changó Fama 


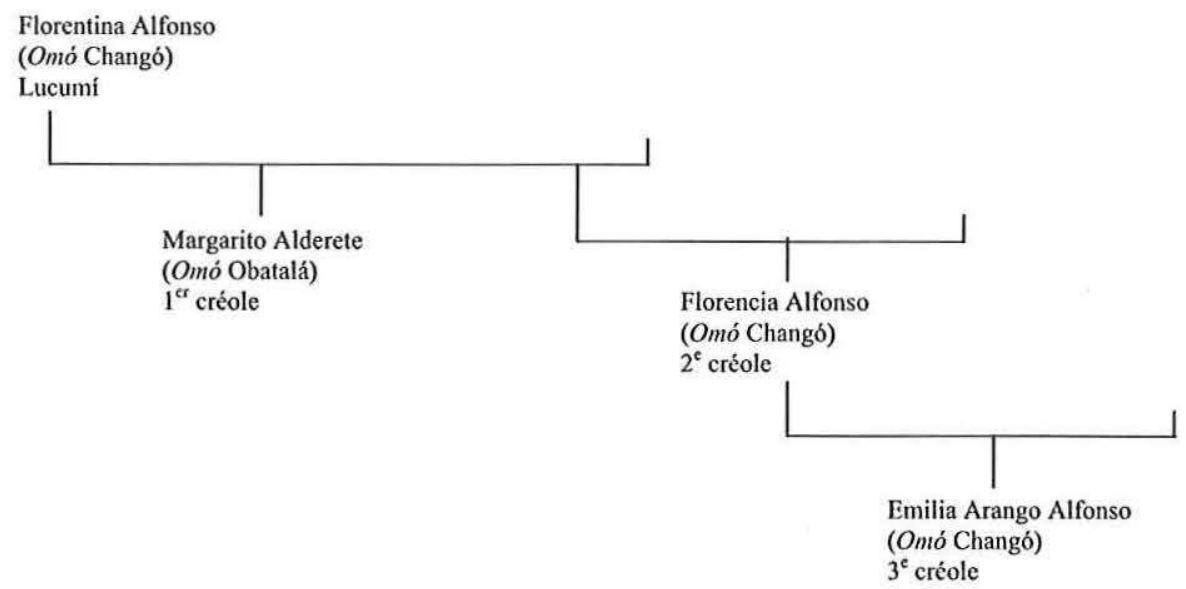

Fig. 2 - Détenteurs de la relique de Changó Fama.

se manifeste dans toutes les sphères de la vie car, d'après eux, «sans Changó Fama, il n'y a pas de famille ".

Nous avons vu que cet héritage africain peut toucher toute une famille. Chez les Arango-Alfonso, plusieurs personnes ont reçu une initiation religieuse individuelle, mais Changó Fama est au centre de la vie familiale. Les festivités des familles héritières d'une relique sont entièrement dédiées au culte en question, parfois même au détriment des fêtes religieuses personnelles. Toute la famille, restreinte et élargie, adore la relique. En effet, le jour de sa fête, on réalise toutes les cérémonies de rigueur, principalement le repas rituel et les tambours (toque) en l'honneur de Changó Fama. La fête de la relique peut être l'anniversaire du jour de sa naissance ou celui du saint catholique avec lequel l'oricha est syncrétisé.

Les reliques sont des pièces fondatrices d'une religiosité qui garde encore ses marques dans la ville. Elles échappent à toute normalisation religieuse, nul besoin d'une quelconque initiation pour devenir héritier d'une relique. Elles restent dans le cadre d'une transmission simple, d'une génération à la suivante, qui se fait de manière " naturelle », sans grands dispositifs ni artifices liturgiques. Cette modalité cultuelle, qui est par définition héréditaire et familiale (par filiation ou par alliance), n'a pas disparu avec l'arrivée de la santería occidentale. Les deux modalités coexistent sans conflit, elles ne sont pas en concurrence car la suprématie des reliques n'est jamais remise en question. 


\section{CONFIGURATION DE LA VILLE DANS LA PÉRIODE POST-ABOLITIONNISTE}

L'urbanisme de Sagua la Grande a été modifié à la suite de l'abolition de l'esclavage en 1868. En effet, les nouveaux hommes libres quittaient les maisons des maîtres et les habitations pour s'installer sur les terrains autorisés à l'extérieur de la ville. L'élargissement du tissu urbain a commencé dès 1874 (Alcover y Beltrán 1905). À cette période, les quartiers de Pueblo Nuevo ${ }^{18}$, Cocosolo et Villa Alegre se sont consolidés. Leur localisation n'a pas été laissée au hasard, les faubourgs naissants devaient être construits sous le vent, c'est-à-dire vers le sud, car le vent souffle de l'est vers le sud/sud-ouest et «l'odeur de nègre » ne devait pas déranger les familles aisées qui étaient installées, justement, à l'est de la ville (dans le quartier qui, aujourd'hui, est appelé Finalet) ${ }^{19}$. À l'est, il n'y avait pas de " quartiers », mais des pavillons et des villas des propriétaires de sucreries ou de chemins de fer; parmi lesquels on comptait quelques familles anglaises. D'ailleurs, l'emplacement du cimetière a été déterminé selon la même conception de l'espace, c'est-à-dire "en orientant les odeurs » pour éviter de perturber certains secteurs.

C'est bien dans ces quartiers consolidés après l'abolition de l'esclavage que fonctionnaient les anciens cabildos et/ou les sociétés de culte afro-cubain. Le plus ancien de tous est le cabildo congo « Kunalumbo », dans le quartier de Pueblo Nuevo, devenu société San Francisco de Asís en 1909. La santería d'origine lucumí n'y a jamais été pratiquée puisque ce cabildo était d'origine congo, néanmoins de nombreux santeros de la ville le fréquentaient ${ }^{20}$. Les trois autres cabildos étaient lucumí. Le cabildo Santa Bárbara du quartier Cocosolo a été déplacé à trois reprises, à chaque fois que la zone où il se trouvait était incorporée au centre ville. Ce cabildo n'a jamais été enregistré comme tel et n'a pas été transformé en société non plus, mais, paradoxalement, c'est bien à travers cette institution que la religion lucumí est entrée dans la ville. Inversement, la société Santa Bárbara du quartier Villa Alegre est née telle quelle en 1898 et, bien qu'on la surnomme " el cabildo de los chinitos », elle n'a jamais existé en tant que cabildo. Depuis, cette société a toujours fonctionné et c'est par elle que sont arrivés à Sagua, dans les années 1950, les tambours batá de fundamento (l'instrument musical par excellence de la santería). La quatrième société, San Miguel Arcángel, située dans le quartier de San Juan, a été enregistrée très tardivement, en 1951, même si elle existait officieusement depuis la fin du Xıx ${ }^{\mathrm{e}}$ siècle. Cette société a été la première à intégrer les modifications introduites dans la santería au $\mathrm{xx}^{\mathrm{e}}$ siècle. Toutes les quatre sont appelées, de façon familière, cabildos. Elles sont situées dans les « quartiers » et ont joué un rôle important dans la transformation du paysage religieux de la ville.

Le « quartier », comme espace urbain différencié et opposé au « centre » de la ville, se maintient jusqu'à la première moitié $\mathrm{du} \mathrm{xx}^{\mathrm{e}}$ siècle. C'est avec la révolution socialiste de 1959 que les deux notions de « quartiers » et de « centre » vont se 
confondre en créant une homogénéité communicationnelle dans le tissu social citadin (Villavicencio, communication personnelle, 1999). Autrefois, les habitants des quartiers n'allaient pas facilement ni fréquemment au centre de la ville (on dit encore " aller au village » pour indiquer le déplacement de la périphérie en direction du centre). Il convient de rappeler que, dans de nombreuses villes du pays, les parcs et les places étaient différemment fréquentés par les Blancs et les Noirs : ces derniers ne pouvaient pas se promener à l'intérieur, qui était exclusivement réservé aux Blancs. La dichotomie quartier/centre reflète, d'une certaine manière, la même conception discriminante d'occupation des espaces publics.

Cet aspect de l'organisation citadine était important pour la vie des cabildos et des sociétés de culte afro-cubain car ils étaient situés dans les «quartiers » et, lorsqu'on apportait le saint patron en procession jusqu'à l'église, c'était bien au centre de la ville que l'on se rendait. Saint François d'Assise du cabildo Kunalumbo (du quartier de Pueblo Nuevo) et sainte Barbe du cabildo de los chinitos (du quartier de Villa Alegre) étaient accompagnés par les tambours consacrés (de fundamento) depuis leur sortie jusqu'au chemin de fer qui se trouvait à proximité. De l'autre côté les attendait la fanfare municipale qui les recevait et les accompagnait en cortège jusqu'à l'église principale puis qui les ramenait, immédiatement, au quartier. Là où la fanfare s'arrêtait de jouer; les tambours reprenaient leur musique jusqu'à leur arrivée aux cabildos. Dans les deux sens, le chemin de fer; interprété comme la limite entre le quartier et le centre, n'était autre chose que la frontière entre "l'africanité » et le "catholicisme » qui se cristallisait dans les espaces urbains. Autrement dit, saint François ou sainte Barbe pouvaient sortir de leur quartier et aller au centre à condition de laisser derrière eux leur « africanité ». Les tambours sonnaient dans le quartier; la fanfare au centre ville, le compromis entre les deux composants des cultes se désolidarisant pour pouvoir traverser la barrière symbolique (le chemin de fer) qui maintenait séparés les deux univers.

Tandis que l'on faisait référence, dans la presse, à certaines sociétés - y compris les sociétés d'entraide des Noirs -, les sociétés afro-religieuses n'étaient jamais mentionnées. Celles-ci sont nées et se sont développées dans l'indifférence de la ville. C'est probablement parce qu'elles ont continué à représenter « l'arriération " en opposition au "progrès » que représentaient les autres sociétés, tendance qui était celle du pays en général ${ }^{21}$. Les sociétés afro-religieuses pouvaient cohabiter avec leurs homologues dans le même espace du quartier, mais il n'y avait pas d'interaction entre elles.

\section{L'ARRIVÉE DES GRANDES TRANSFORMATIONS}

Les années suivant l'abolition de l'esclavage, c'est-à-dire après 1886, ont été riches en changements de toutes sortes. La condition récemment acquise d'hom- 
mes libres permettait aux anciens esclaves de se déplacer à l'intérieur de l'île, ce qui était impensable par le passé. Malgré cela, dans la région centrale, il n’y a pas eu d'évolutions significatives dans la composition de la population. Néanmoins, Rebecca Scott (2001) affirme que Sagua la Grande a été le lieu choisi pour l'" exil interne » de certains anciens esclaves, à travers l'implantation de communautés indépendantes en milieu rural, mais sans que ce modèle se propage dans d'autres régions du pays.

Deux ans après l'abolition de l'esclavage, en 1888, se sont produits de grands événements qui ont été à l'origine de la première grande transformation dans le processus de construction des pratiques religieuses d'origine africaine à Sagua. Les trois faits qui ont modifié complètement le paysage religieux sont : l'interdiction de la sortie à la Fête des Rois, la consolidation du spiritisme et l'introduction de la santería havanaise. Ces trois événements jettent les bases d'un nouveau fonctionnement religieux qui régira tout le $\mathrm{xx}^{\mathrm{e}}$ siècle.

L'interdiction tardive - notamment par rapport à celle de La Havane qui date de 1884 - de la sortie des cabildos de Sagua, lors de la Fête des Rois, est rapportée par l'historien local comme un grand fait de « civilisation et culture » :

Le premier acte curieux et transcendantal de l'année 1888 a sans doute été l'arrêté du maire [Felipe Obeso y Robles], daté du 4 janvier, interdisant le défilé des traditionnelles comparses ou cabildos de Rois dans les rues de la ville. Le maire justifiait cette décision par le fait que la cohue pouvait représenter un moyen de propagation d'une épidémie de variole qui sévissait dans la ville. Un autre argument, non moins suggestif, était que les cabildos contrastaient avec le [haut] degré de civilisation atteint par la race noire. Mesure sympathique et progressiste, digne d'être saluée, parce que rien ne peut sembler plus sauvage que ces cabildos africains qui caractérisent à Cuba les fêtes du Jour des Rois. (Alcover y Beltrán 1905, pp. 388-389)

Cette mesure gouvernementale a freiné le dynamisme de la vie religieuse des Africains et de leurs descendants et favorisé leur repli sur eux-mêmes. Si la mesure visait à sortir de la scène publique les fêtes des Noirs, en revanche elle avantageait d'autres collectivités dont, bien entendu, les Blancs. Dans la même année, 1888, a eu lieu la réunion des Catalans au théâtre Lazcano avec un défilé de charrettes, une chorale et la parade à travers la ville. L'idéologie du "blanchiment ", sous-bassement de la transformation sociale, se manifestait aussi dans les expressions urbaines des groupes ethniques qui formaient la nation naissante.

Par ailleurs, un nouveau mouvement religieux se consolidait : le spiritisme. Son introduction dans l'île était alors récent ${ }^{22}$, et encore plus à Sagua. Une année plus tôt, le 15 juin 1887, la revue $L a$ Alborada ${ }^{23}$ affirmait que la doctrine spirite était connue depuis peu dans la ville et ne comptait que quatre ou six pratiquants. L'importance de cette donnée n'est pas négligeable si nous envisageons qu'aujourd'hui le spiritisme est inséparable de la pratique de la santería. À ce moment-là, le spiritisme fonctionnait dans le centre ville et ses adeptes étaient 
tous des Blancs, alors que la santería arrivait à peine et s'installait dans les quartiers périphériques parmi les Africains et leurs descendants. La même année, une commission du centre spirite de Sagua, appelé « El Salvador », participait au Congrès international spirite de Barcelone ; juste après, ces mêmes personnes se sont rendues à Paris pour visiter le tombeau d'Allan Kardec au cimetière du Père Lachaise et y ont déposé une œuvre artistique avec le nom du centre et de la ville de Sagua la Grande (Alcover y Beltrán 1905). En même temps, la revue $L a$ Alborada publiait le texte complet du discours de Camille Flammarion prononcé à la mort du fondateur du spiritisme kardeciste, décédé en 1869. Dès ses débuts, cette publication diffusait les écrits du penseur parisien ainsi que ceux d'Alfred Russell Vallace de Nantes et de José Buchanam de Londres, du Journal of Man, ce qui montre bien les liens que la revue entretenait avec ses homologues européens ${ }^{24}$.

Le troisième grand événement, survenu en 1888, selon la mémoire collective, et sans doute le plus significatif, est la première initiation à la Regla de Ocha, pratique jusque-là méconnue dans la ville. Cet événement sera le premier pas de l'expansionnisme lucumí occidental dans la région de Sagua.

\section{LE SANTO À L'AFricAINE}

La santería ou Regla de Ocha, en tant que religion « normée ", s’implante à Sagua par le biais d'un cabildo, celui de Santa Bárbara, dans le quartier périphérique de Cocosolo (Figure 3). C'est au siège de ce cabildo que deux femmes africaines, Ma' Antoñica Wilson et Ma' Joaquina Mora, furent les premières à être initiées, en 1888, à la religion lucumí occidentale. Tout paraît indiquer qu'elles étaient d'origine lucumí, surtout Ma' Antoñica Wilson qui, d'après ceux qui l'ont connue, avait le visage rayé de trois traits verticaux sur chaque joue, marque des esclaves en provenance d'Afrique de l'Ouest (Deschamps Chapeaux 1969, p. 67).

Les deux femmes étaient des filles d'Ochún, la déesse des eaux douces. La première reçut pour nom liturgique Ochún Funké et la seconde Ba Mefún. La Regla de Ocha a été introduite à Sagua par le babalao ${ }^{25}$ havanais Remigio Herrera - un grand nom, mieux connu sous son appellation religieuse d'Addechina ${ }^{26}$, puisqu'il aurait, selon les anciens de la ville, présidé les cérémonies - et par un santero de Cárdenas (province de Matanzas), appelé Trinidad Infante. Difficile de savoir lequel des deux a dirigé les initiations, d'autant plus que c'est une tâche liturgique réservée aux santeros, mais en tout cas le nom d'Addechina est resté étroitement lié à l'arrivée de la santería à Sagua ${ }^{27}$.

De l'initiation de ces deux femmes africaines, il ne reste, bien évidemment, aucun souvenir. Néanmoins, tous les religieux de la ville - surtout les plus vieux leur accordent une place de pionnières en matière de santería. Ma' Antoñica 


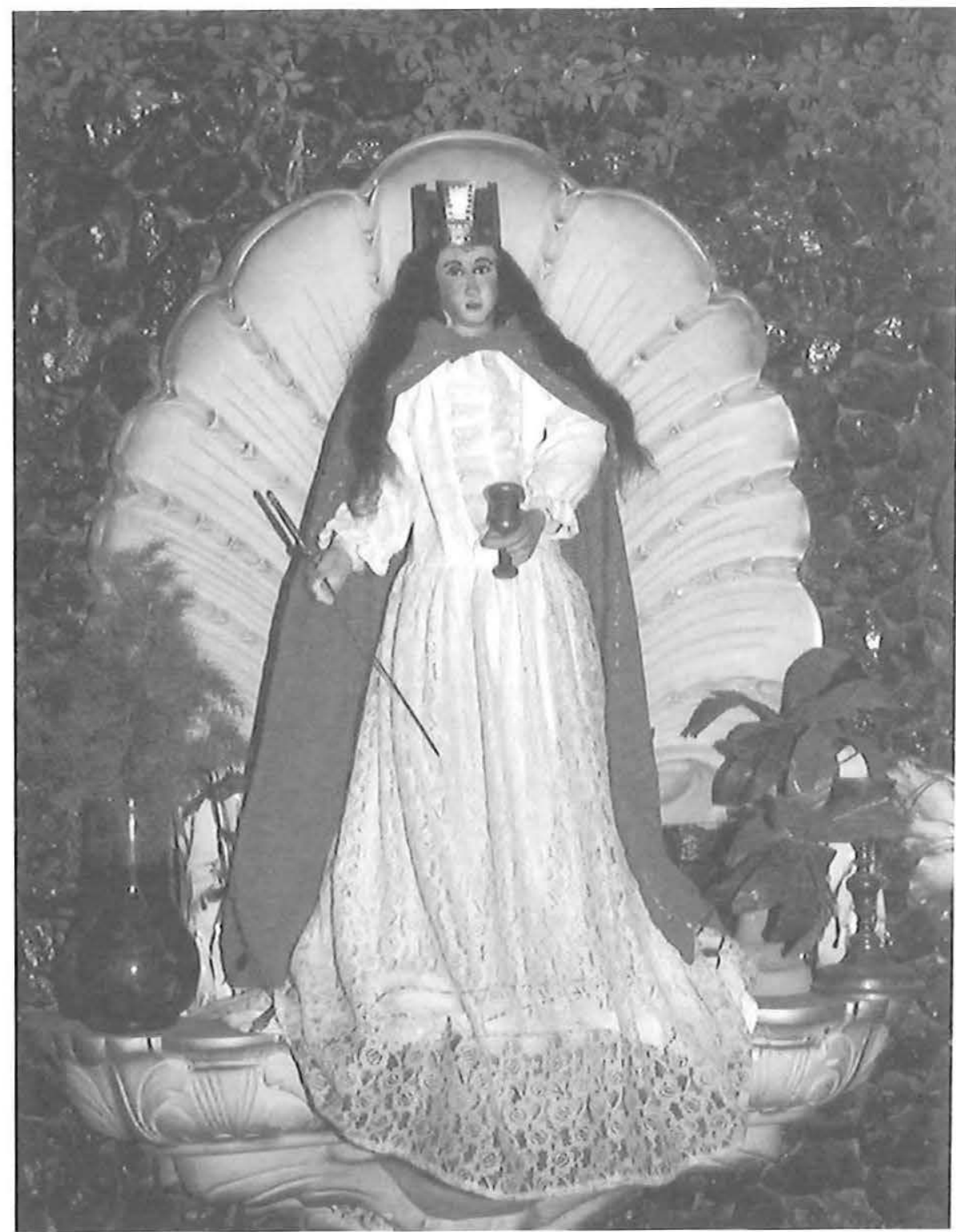

Fig. 3 - Statue de sainte Barbe du cabildo Santa Bárbara du quartier de Cocosolo (cliché Testa). 
Wilson ${ }^{28}$ a eu une importante descendance religieuse et a occupé jusqu'à sa mort la direction du cabildo de Santa Bárbara à Cocosolo. En revanche, Ma' Joquina Mora a constitué une grande famille biologique de laquelle est issu le seul babalao que compte Sagua aujourd'hui, Rolando Mora García, son arrière-petit-fils.

Bien que la santería occidentale ait connu un développement dans le quartier de Cocosolo, la zone par excellence de l'afro-religiosité est celle de San Juan, espace géographique où l'on trouve les reliques de famille. Là aussi, une femme est restée dans la mémoire collective locale et a été considérée comme la grande santera du début du $\mathrm{xx}^{\mathrm{c}}$ siècle : Elena Mestre, mieux connue sous le sobriquet de "Nena ». Nous n'avons aucune information sur les liens religieux entretenus entre les deux femmes africaines et Elena Mestre, mais vraisemblablement on peut penser que ces liens ont été rendus possibles grâce à Félix Laserie, surnommé Lucumí, membre du cabildo Santa Bárbara de Cocosolo, car, à la mort des Africaines du cabildo, il aurait déménagé chez Elena Mestre. D'ailleurs, Agustina Thondike (1909-2002), sa seule "filleule ${ }^{29}$ (ahijada), encore vivante au moment de notre enquête, avait été initiée conjointement par Elena Mestre et Félix Laserie, le 8 juillet 1926, dans le quartier de San Juan. Dans les dernières années du $\mathrm{XIX}^{\mathrm{e}}$ siècle et les premières du $\mathrm{Xx}^{\mathrm{c}}$, se pratiquait à Sagua le "santo à l'africaine " (santo a la africana). Cette expression est liée au fait que, pour les anciens de la ville, cette modalité cultuelle avait été introduite par des Africains (Adecchina et Trinidad Infante) venus de La Havane et de Matanzas. Dorénavant, la pratique religieuse devient individuelle et, à la différence de celle des reliques de famille, plusieurs orichas peuvent cohabiter dans la même maison, chacun correspondant à une seule et même personne. De plus, et pour la première fois, le processus initiatique est mis en place pour devenir pratiquant car, dans les cultes de type familial, il n'y avait pas de recours à l'initiation. L'initiation impliquait un temps de réclusion (dans le cas d'Agustina, il a duré douze jours), la coupe rituelle des cheveux, même si la tête devait demeurer toujours couverte, et le port de vêtements blancs dans l'année qui suivait l'entrée en religion (iyaworage ${ }^{30}$ ). Un autre aspect important de ce processus initiatique était la remise de pièces rituelles :l'initié recevait la représentation matérielle de sa divinité tutélaire ainsi que les santos guerreros (Eleguá, Ogún, Ochosi et Osun). Cette variante est encore appelée « pieds et tête " ( pié y cabeza), les pieds étant les santos guerreros et la tête, l'ange gardien ou oricha tutélaire. Â partir du moment où la personne avait son santo ${ }^{31}$ chez lui, sa maison devenait alors « la maison de... (Changó, Ochún, etc.) ». Cela signifiait que si, par exemple, quelqu'un (déjà initié ou non) avait besoin de "faire une œuvre » ${ }^{32}$ auprès d'un autre santo que le sien, il était obligé de s'adresser au santero qui avait chez lui cette divinité. L'oricha tutélaire ayant une exclusivité presque absolue sur ses fils, il était nécessaire de passer par l'un d'eux pour avoir accès à la divinité afin d'accomplir l'activité rituelle.

Agustina Thondike se rappelait aussi d'autres cérémonies faites par sa marraine, notamment celles réalisées en pleine nature. D'après Agustina, son initia- 
tion n'a pas commencé par le bain lustral dans le fleuve, comme c'est l'habitude de nos jours. Mais les eaux douces de Sagua ont joué un rôle prépondérant dans cette maison de culte : chaque 24 juin, jour du saint patron du quartier (saint Jean), Nena Mestre faisait plonger dans le fleuve tous les filleuls qui avaient besoin d'une " purification ». Pour les autres, elle emportait de l'eau dans une bassine qu'elle mettait devant l'autel, pour qu'ils se lavent avant de saluer le santo. Mais, bien qu'Elena Mestre ait été connue pour ses grandes connaissances et ses pouvoirs religieux, ayant de surcroît initié beaucoup de personnes à Sagua, elle n'a jamais été reconnue santera. En effet, quelques-uns des filleuls d'Elena Mestre, surtout ceux qui ont consacré leur vie à la santería, sont allés à La Havane pour " refaire leur santo », c'est-à-dire accomplir une deuxième initiation afin d'être reconnus comme santeros. Cela explique une phrase que l'on entend souvent à Sagua : «Un tel a fait santo deux fois ». Mais s'agissait-il d'une répétition de l'initiation antérieure ou d'une initiation complètement nouvelle? Les avis des anciens de la ville sont partagés. À l'instar de Minga (filleule de baptême d'Elena Mestre et «filleule » d'initiation dans la santería de Nenene, elle-même «filleule » d'Elena Mestre), certains pensent qu'il s'agissait d'une confirmation de la première initiation à travers un processus semblable à celui que l'on réalise pour l'enfant dont la mère a été initiée lorsqu'elle était enceinte, c'est-à-dire qu'il la « reçoit » dans le ventre et la « confirme » après la naissance. Pour d'autres, comme Chelé (deuxième santero en importance dans la ville ${ }^{33}$ ), lors des initiations faites par Elena Mestre, il n'y aurait pas eu de fixation de l'oricha tutélaire dans la tête de l'initié, autrement dit cela correspondait à une demi initiation ; en termes vernaculaires il s'agirait d'un "santo lavé » (santo lavado) et non pas d'un «santo couronné » (santo coronado), expression qui désigne l'initiation proprement dite. Chacune des deux réponses renvoie toujours à l'idée d'une non-reconnaissance du santo à l'africaine, autrement dit à la caducité de cette modalité de culte à partir de l'année 1927.

Ce même type de phénomène existe dans d'autres villes de Cuba, notamment à Matanzas. La raison se trouverait dans l'histoire des esclaves lucumís sur le sol cubain. En effet, les Lucumís sont connus à Cuba depuis le XVII ${ }^{\mathrm{e}}$ siècle mais, à l'époque, ils ne constituaient qu'un faible pourcentage de la population esclave. Leur arrivée en masse s'est faite au XIX ${ }^{\mathrm{e}}$ siècle, à la suite de la chute de l'empire d'Oyo ${ }^{34}$. Ainsi, vers 1850 , ils représentaient $35 \%$ du total des esclaves africains. D’après Miguel Ramos (2003), les Lucumís, entrés jusqu'aux premières années du $\mathrm{XIX}^{\mathrm{e}}$ siècle, seraient originaires d'Egbado, tandis que ceux arrivés par la suite viendraient d'Oyo. Or chaque territoire avait sa propre tradition religieuse. Ainsi, à Cuba, se sont développées deux pratiques cultuelles d'origine lucumí, évoluant différemment selon les époques. La plus ancienne, celle d'Egbado, aurait dominé le culte lucumí approximativement jusqu'en 1825. De cette tradition découlent les deux variantes (celle des «reliques de famille» et celle du «santo à l'africaine ${ }^{35}$ ) que nous avons déjà évoquées dans le cas de Sagua. Ensuite, avec 
l'arrivée des esclaves lucumís originaires d'Oyo, l'imposition de leur propre mode d'initiation a fait disparaître la tradition d'Egbado. Cela expliquerait la nonreconnaissance de la modalité « pieds et tête » ou « santo à l'africaine » et, donc, l'obligation pour certains santeros de Sagua de refaire leur initiation.

L'expression "santo à l'africaine» acquiert encore plus de force lorsque la nouvelle mode arrive de La Havane, à partir de 1927, car elle met hors jeu les initiations pratiquées avant cette date. Le «santo à l'africaine» a fortement marqué la religiosité locale, la grande innovation étant celle du passage d'un culte familial, ancré dans le lignage biologique et sans procédure initiatique (la relique de famille), à un culte individuel, inséré dans un lignage rituel, auquel on est intégré à la suite d'une initiation (santo à l'africaine). Par ailleurs, les femmes, d'abord africaines, ensuite créoles, avaient un grand pouvoir avant 1927, ce qui n'est plus le cas après cette date.

\section{Le SANTO À LA CUBAINE}

Le fonctionnement religieux que nous venons d'analyser commence à perdre de la vigueur avec l'arrivée de la nouvelle «mode » havanaise. En fait, il n'a été valable qu'entre 1888 et 1927 . Nous avons dit que certains pratiquants de la ville ont refait leur initiation. Le premier à l'accomplir à nouveau a été Valentín Alfonso, président de la société San Miguel Arcángel du quartier de San Juan, là où se trouvent les reliques de famille. Bien qu'il ait fréquenté assidûment la maison d'Elena Mestre et se soit considéré comme un de ses « filleuls», il avait reçu son « santo à l'africaine » de Pablo González à Recreo, dans la province de Matanzas. Cette initiation dans le culte d'Oyá, son oricha tutélaire, n'a pas été reconnue quand il est arrivé à La Havane ${ }^{36}$. Il a dû y retourner pour faire sa nouvelle initiation, cette fois-ci, en suivant la modalité cubaine, également appelée « santo créole » (santo criollo). À cette occasion, il se mit en relation avec une maison de culte renommée, dans le quartier havanais de Guanabacoa. Son parrain était José Urquiola, Echubbi, mieux connu par son surnom " pata 'e palo », et sa deuxième marraine (oyugbona ${ }^{37}$ ), Francisquita Estensi, Chango Bolé, fondatrice du cabildo San Roque de Palmira (à côté de la ville de Cienfuegos). Sa deuxième initiation a eu lieu le 27 mai 1927. Bien que la première fût d'Oyá, cette fois-ci Changó n'a pas voulu céder son fils et l'a réclamé en exclusivité. Ainsi, Valentín Alfonso est devenu omó Changó ${ }^{38}$, avec le nom rituel d'Addé Kola, traduit comme " roi de la couronne ».

Mais le chemin ouvert par Valentín Alfonso dans la santería créole n'a pas rapidement fait des émules dans la ville de Sagua. Il a fallu attendre treize ans pour qu'une autre personne suive ses pas. Le 29 mai 1940, Juan García Fonseca, mieux connu sous le nom de "Bienvenido", dont le nom rituel était Omi Taguardé, a été initié au culte de Yemayá par sa «marraine » ${ }^{39}$, María La O 
Batte (Orfandei), dans le quartier havanais de Puentes Grandes, chez sa deuxième marraine (oyugbona) Bernardina Pérez (Ochún Funké), de surnom « Nina La Puente ». L'obba-oriaté ${ }^{40}$ de l'initiation était Octavio Samá, mieux connu sous son nom religieux d'Obbadimeyi, disciple direct de Latuán, femme lucumí pionnière de la tradition d'Oyo à Cuba. À Sagua, Juan García Fonseca n'appartenait à aucun cabildo ou autre société, mais il habitait le quartier de Pueblo Nuevo, à proximité de Kunalumbo, le cabildo congo, dont il était un habitué. Son amitié avec Catalina Prendes, femme congo et ancienne reine du cabildo, était connue de tous à tel point qu'elle a même été inscrite dans les chansons de l'institution afro-cubaine ${ }^{41}$. Juan García Fonseca a aussi été la première personne blanche de Sagua à s'initier dans la santería. Comme beaucoup d'autres santeros de la ville, il n'a pas eu d'enfants, mais il a créé une grande famille religieuse. Il a d'ailleurs lui-même initié la deuxième personne de Sagua, Jesús López Zúñiga, omó Eleguá, le 16 juillet 1942, dans la maison havanaise où il avait reçu son initiation. Aujourd'hui, Jesús López Zúñiga ${ }^{42}$ est le santero le plus important (santero mayor) de la province de las Villas.

La même année, c'est Valentín Alfonso qui amène le « santo à la cubaine » à Sagua la Grande. Au siège de la société, San Miguel Arcángel, qu'il présidait, il a initié, le 12 novembre 1942, cinq nouveaux santeros dont il ne reste que l'aîné, Pedro Pablo Dreke Alfonso ${ }^{43}$, « Chelé », omó Obatalá ${ }^{44}$. Pour cette célébration, il a fait venir des santeros de La Havane, Matanzas, Colón, Jovellanos, Cárdenas et Perico, la présence de ce grand nombre de religieux offrant des témoins à ces initiations. Il y a eu plusieurs oyugbonas, dont la célèbre havanaise, du quartier de Regla, Dominga Sandoval, omó Changó, qui avait pour nom rituel Obá Tuké. Valentín Alfonso est donc devenu parrain de cinq nouveaux santeros.

Bien que nous ne connaissions pas qui était l'obbá-oriaté de l'initiation de Valentín Alfonso, nous savons en revanche qu'il a été secondé, lors de l'initiation de ses premiers filleuls, par Tomás Romero ${ }^{45}$, disciple direct d'Obbadimeyi. Par ailleurs, Obbadimeyi lui-même a été l'obbá-oriaté de «Bienvenido » et de son filleul Jesús López Zúñiga. Donc, c'est bien la lignée religieuse de l'obbasaoriaté $^{46}$ Latuán, c'est-à-dire la tradition d'Oyo, qui a été introduite à Sagua à partir de 1927. C'est en effet l'arrivée de cette tradition qui a annulé celle d'Egbado, ce qui expliquerait la non-reconnaissance du « santo à l'africaine » et donc l'initiation à la modalité nommée «créole » ou « santo à la cubaine » à Sagua. Paradoxalement, la modalité dite "plus pure», associée au symbole majeur de la culture yoruba - Oyo-, devient le «santo à la cubaine» ou " créole".

Nous avons déjà vu qu'à l'époque où l'on pratiquait le « santo à l'africaine » le novice recevait la représentation matérielle de son oricha tutélaire (ou « tête ») et les santos guerreros (ou « pieds »). Désormais, avec l'introduction de la nouvelle mode havanaise, l'initié recevra, en plus des précédents, tout un ensemble d'orichas du panthéon lucumí, dont les quatre divinités de base sont Obatalá, 
Changó, Yemayá et Ochún. Il est intéressant de signaler que la première initiation à la santería créole à Sagua, en 1942, coïncide avec la sortie du livre El sistema religioso de los afrocubanos de Rómulo Lachatañeré, livre dans lequel il est question de la notion de " panthéon lucumí », c'est-à-dire un ensemble de divinités ${ }^{47}$. De plus, ce nouveau mode d'initiation veut que chaque oricha soit reçu dans toute sa matérialité. Auparavant, le novice ne recevait que les pierres réceptacles des orichas et, dans quelques cas, une figure anthropomorphe en bois représentant la divinité en question. Désormais, l'impétrant reçoit aussi les cauris ${ }^{48}$ et les outils (herramientas) de chaque oricha ; il est tenu de respecter l'iyaworage pendant une année entière et, durant l'initiation, a lieu une longue séance oraculaire appelée itá, avec les prescriptions et les interdictions que le santero devra observer le reste de sa vie. La durée de l'initiation est officialisée également et se réduit systématiquement à sept jours, alors qu'avant elle était bien plus longue : douze jours pour le cas d'Agustina et encore plus pour d'autres. De toute évidence, nous sommes en présence d'une nouvelle définition du profil santero, beaucoup plus complexe et, surtout, assez codifié. Nous verrons aussi qu'à partir de ce moment la pratique isolée de la santería n'est plus possible : pour toute nouvelle initiation, d'autres santeros doivent être présents afin d'en être les " témoins ».

À l'arrivée du "santo à la cubaine ", les liens qui se tissaient entre les pratiquants de Sagua et ceux de la capitale ne se résumaient pas à des relations « parrain »-« filleul ». En effet, l'engagement allait bien plus loin, notamment dans la vie associative. La sociedad San Miguel Arcángel, qui a toujours été « la maison de Valentín Alfonso ", est devenue le lieu de nombreuses initiations. Un des santeros havanais qui fréquentait cette maison de culte de manière assidue était Gregorio Torregrosa (Obbá Bí), omó Changó. Il y allait comme oflíciant de la liturgie locale, en tant qu'obbá-oriaté, mais a aussi effectué la confirmation des pratiquants de la ville, initiés par Nena Mestre à la manière africaine : cela a été le cas de Gregoria Alfonso (Ogún Bí), surnommée « Nena Zacarías », omó Ogún, dont les « parrains » ont été Gregorio Torregrosa et Dominga Sandoval, la même santera havanaise qui a été la deuxième marraine (oyugbona) des premiers initiés à Sagua en 1942. Un cas similaire est celui de Deogracia Herrera (Arokumatindé ${ }^{49}$ ), « Nenene ", omó Changó, qui a confirmé, de nouveau, son santo avec Torregrosa, sa oyugbona étant Gregoria Alfonso. L'obbá-oriaté des deux femmes a également été Tomás Romero, lui-aussi Havanais. Ainsi, en confirmant le santo avec Gregorio Torregrosa, les nouveaux santeros devenaient automatiquement ses « filleuls ».

Gregorio Torregrosa a continué pendant longtemps à se rendre à Sagua en qualité d'obbá-oriaté, dirigeant les cérémonies de l'initiation de Felicia Mestre et d'Irene Herrera en 1947, toutes les deux « filleules » de Gregoria Alfonso. Felicia avait rencontré sa future marraine chez Elena Mestre, la pionnière, dans le même quartier de San Juan. Après leur initiation, les deux femmes furent présentées au 


\begin{tabular}{|c|c|c|c|c|c|c|c|}
\hline Santero & $\begin{array}{l}\text { Initiation à } \\
\text { l'africaine }\end{array}$ & $\begin{array}{l}\text { Initiation } \\
\text { à la } \\
\text { cubaine }\end{array}$ & Date & $\begin{array}{l}\text { Marraine } \\
\text { ou parrain }\end{array}$ & Oyugbona & \begin{tabular}{|l} 
Lieu \\
d'initiation
\end{tabular} & $\begin{array}{l}\text { Obbá- } \\
\text { Oriaté }\end{array}$ \\
\hline \multirow[t]{2}{*}{$\begin{array}{l}\text { Valentín } \\
\text { Alfonso }\end{array}$} & $\mathrm{X}$ & & & $\begin{array}{l}\text { Pablo } \\
\text { González }\end{array}$ & & \begin{tabular}{|l}
$\begin{array}{l}\text { Recreo } \\
\text { (Matanzas) }\end{array}$ \\
\end{tabular} & \\
\hline & & $\mathrm{X}$ & 1927 & $\begin{array}{l}\text { José } \\
\text { Urquiola }\end{array}$ & $\begin{array}{l}\text { Francisquita } \\
\text { Estensi }\end{array}$ & $\begin{array}{l}\text { Regla } \\
\text { (La Havane) }\end{array}$ & \\
\hline $\begin{array}{l}\text { Juan García } \\
\text { Fonseca }\end{array}$ & & $\mathrm{X}$ & 1940 & $\begin{array}{l}\text { María } \\
\text { La O Batte }\end{array}$ & $\begin{array}{l}\text { Bernardina } \\
\text { Pérez }\end{array}$ & $\begin{array}{l}\text { Puentes } \\
\text { Grandes } \\
\text { (La Havane) } \\
\end{array}$ & Obbadime yi \\
\hline $\begin{array}{l}\text { Jesús López } \\
\text { Zúñiga }\end{array}$ & & $\mathrm{X}$ & 1942 & $\begin{array}{l}\text { Juan García } \\
\text { Fonseca }\end{array}$ & $\begin{array}{l}\text { Bernardina } \\
\text { Pérez }\end{array}$ & $\begin{array}{l}\text { Puentes } \\
\text { Grandes } \\
\text { (La Havane) } \\
\end{array}$ & Obbadime yi \\
\hline $\begin{array}{l}\text { Pedro Pablo } \\
\text { Dreke }\end{array}$ & & $X$ & 1942 & $\begin{array}{l}\text { Valentín. } \\
\text { Alfonso }\end{array}$ & $\begin{array}{l}\text { Dominga } \\
\text { Sandoval }\end{array}$ & \begin{tabular}{|l|} 
Quartier San \\
Juan (Sagua) \\
\end{tabular} & $\begin{array}{l}\text { Tomás } \\
\text { Romero }\end{array}$ \\
\hline \multirow[t]{2}{*}{$\begin{array}{l}\text { Gregoria } \\
\text { Alfonso }\end{array}$} & $\mathrm{X}$ & & & $\begin{array}{l}\text { Elena } \\
\text { Mestre }\end{array}$ & & \begin{tabular}{|l|} 
Quartier San \\
Juan (Sagua) \\
\end{tabular} & \\
\hline & & $\mathrm{X}$ & 1947 & $\begin{array}{l}\text { Gregorio } \\
\text { Torregrosa }\end{array}$ & $\begin{array}{l}\text { Dominga } \\
\text { Sandoval }\end{array}$ & La Havane & $\begin{array}{l}\text { Tomás } \\
\text { Romero }\end{array}$ \\
\hline \multirow[t]{2}{*}{$\begin{array}{l}\text { Deogracia } \\
\text { Herrera }\end{array}$} & $\mathrm{X}$ & & & $\begin{array}{l}\text { Elena } \\
\text { Mestre } \\
\end{array}$ & & \begin{tabular}{|l|} 
Quartier San \\
Juan (Sagua) \\
\end{tabular} & \\
\hline & & $x$ & 1947 & $\begin{array}{l}\text { Gregorio } \\
\text { Torregrosa }\end{array}$ & $\begin{array}{l}\text { Gregoria } \\
\text { Alfonso }\end{array}$ & La Havane & $\begin{array}{l}\text { Tomás } \\
\text { Romero }\end{array}$ \\
\hline Felicia Mestre & & $X$ & 1947 & $\begin{array}{l}\text { Gregoria } \\
\text { Alfonso }\end{array}$ & $\begin{array}{l}\text { Deogracia } \\
\text { Herrera }\end{array}$ & $\begin{array}{l}\text { Quartier San } \\
\text { Juan (Sagua) }\end{array}$ & $\begin{array}{l}\text { Gregorio } \\
\text { Torregrosa }\end{array}$ \\
\hline Irene Herrera & & $x$ & 1947 & $\begin{array}{l}\text { Gregoria } \\
\text { Alfonso }\end{array}$ & $\begin{array}{l}\text { Deogracia } \\
\text { Herrera }\end{array}$ & \begin{tabular}{|l|} 
Quartier San \\
Juan (Sagua) \\
\end{tabular} & $\begin{array}{l}\text { Gregorio } \\
\text { Torregrosa }\end{array}$ \\
\hline Dominga Pérez & & $\mathrm{X}$ & 1959 & $\begin{array}{l}\text { Deogracia } \\
\text { Herrera }\end{array}$ & $\begin{array}{l}\text { Gregoria } \\
\text { Alfonso }\end{array}$ & $\begin{array}{l}\text { Quartier San } \\
\text { Juan (Sagua) }\end{array}$ & \\
\hline
\end{tabular}

FIG. 4-Récapitulatif des premières initiations à Sagua, modalités à l'africaine et à la cubaine.

tambor $^{50}$ dans le cabildo de Yemayá de Pepa, Echú Bí, à Regla, car cette femme avait été la "marraine» de Gregorio Torregrosa, c'est-à-dire qu'elle était l'arrière-grand-mère de santo des deux iyawós. Pepa était la fille biologique d'Addechina, le babalao havanais qui avait amené le «santo à l'africaine " à Sagua en 1888 (Figure 4).

Mais Gregorio Torregrosa n'a pas été seulement un santero très reconnu pour ses qualités religieuses, il était également le président de la «sociedad Culto Lucumí San Pedro Crisólogo » de La Havane ${ }^{51}$. La société fut fondée le 2 mars 1937 et, selon sa charte ${ }^{52}$, elle était composée d'« individus cubains qui pratiquent la religion lucumí ». Le but principal de cette société était d'« offrir de l'aide morale et matérielle à ses associés ", ce qui rappelle l'assistance que les cabildos de nación $\mathrm{du} \mathrm{XIX}{ }^{\mathrm{e}}$ siècle fournissaient à leurs membres. Or la fonction de cette société allait plus loin. Elle jouait un rôle primordial dans la normalisation de la santería : dans son article 7, la charte signale que « la consécration et l'initiation des associés seront vérifiées dans les locaux de l'association et à une date fixée par le conseil d'administration ". Cette volonté normalisatrice qui animait la société se matérialisait par un diplôme octroyé à chaque membre (Figure 5). 


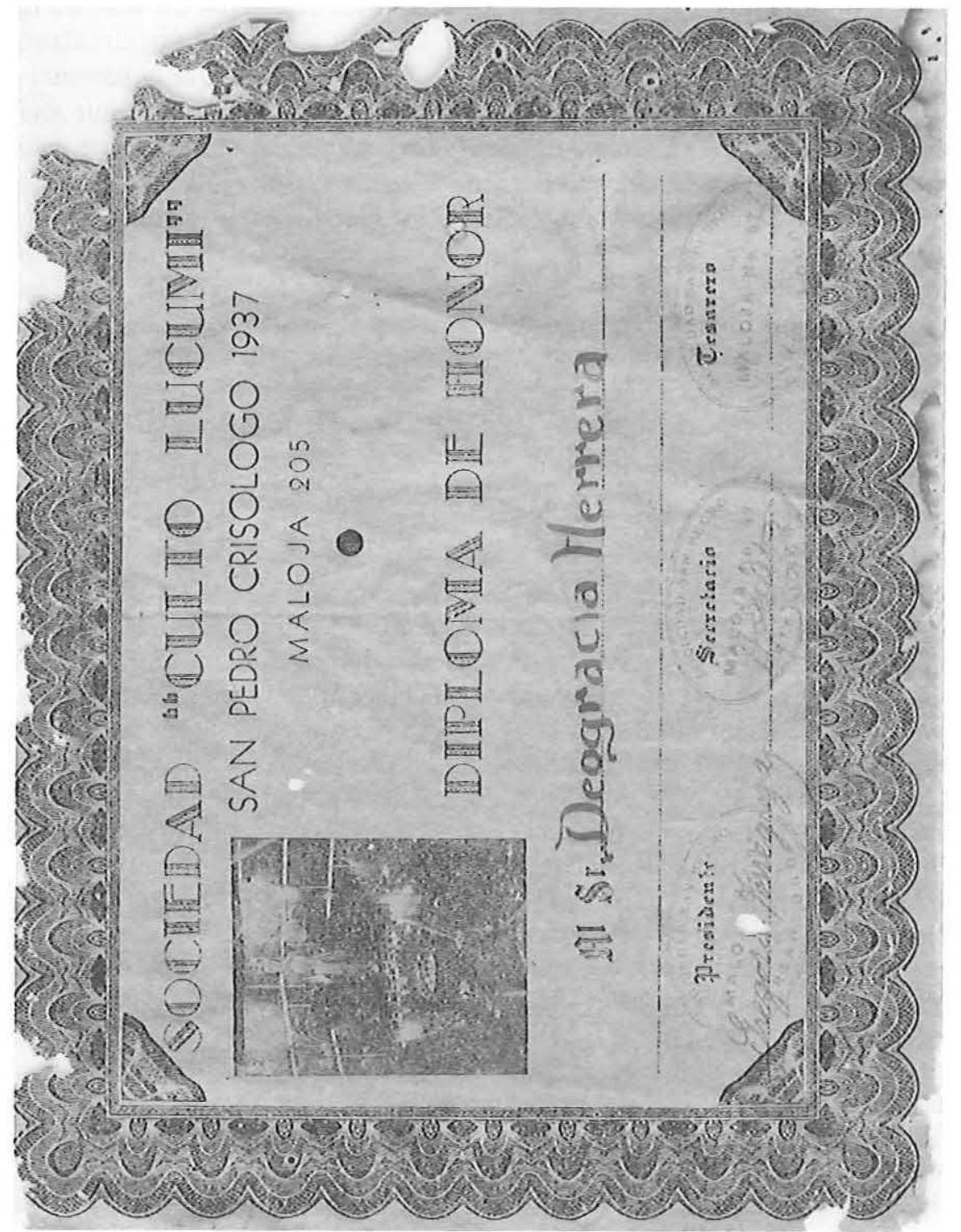

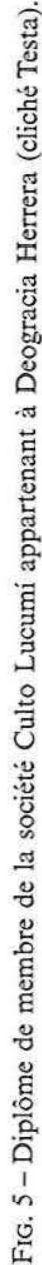


Mais, les sociétés n'étaient pas l'unique mécanisme de contrôle dont la santería occidentale usait dans son processus expansionniste. Il y avait aussi le monopole des tambours batá de fundamento, appelés aussi Añá du nom de la divinité qui y réside. Fernando Ortiz (1994, p. 144) fait référence à une circulaire adressée aux santeros en 1950 qui signale, dans une note finale, que la présentation d'un iyawó n'aura pas de valeur si elle ne se fait pas devant un tambour Añá reconnu à la Havane et à Matanzas. La circulaire est signée par quatre grands omó $A \tilde{n} a{ }^{53}$ havanais dont le réputé Pablo Roche. Comme à Sagua, il n'y a pas eu de tambours Añá jusque dans les années 1950, les santeros étaient obligés de se présenter dans d'autres villes et, selon ce que racontent les plus anciens, beaucoup d'entre eux sont morts sans jamais avoir été présentés au tambour. D'ailleurs, les premiers tambours Añá de Sagua appartiennent à la société Santa Bárbara de Villa Alegre et ont été faits par Miguel Alsina à Matanzas ${ }^{54}$. Ces tambours ont eu le monopole de la musique religieuse de la ville pendant quatre décennies. Ce n'est que dans les années 1990 qu'a été fabriqué le deuxième jeu de tambours consacrés.

\section{Conclusion}

Nous avons vu que les cabildos et les sociétés de Sagua sont les lieux où la santería a pris place dans la ville, à travers ses deux modalités, africaine et créole. Les responsables des cabildos ou des sociétés sont devenus à leur tour les « marraines » et les « parrains » des pratiquants de la ville. Aujourd'hui, à Sagua, il y a de nombreux conflits entre les santeros et santeras des différentes maisons de culte, héritières ou non des anciennes associations. Autrefois, les liens étaient bien plus harmonieux et les rapports se traduisaient surtout par l'entente et la collaboration. Nous avons de cela maints exemples, comme le cas de la sacralisation des statues vénérées dans les cabildos et les sociétés. La statue de saint Michel Archange, de la société qui porte le même nom, est arrivée en 1931, probablement d'Espagne. Elle est d'abord passée par l'église locale, où elle a été baptisée par le curé, les parrains étant Luis Chis (président de la société Santa Bárbara de Villa Alegre) et Anastasia Arango. Ensuite, elle a été apportée au siège de la société du quartier de San Juan, où elle a subi les mêmes rituels qu'une personne que l'on initie, ces cérémonies étant dirigées par le même Valentín Alfonso.

Les cabildos et les sociedades ont été des lieux d'initiation qui ont suivi tour à tour les modalités exportées de la région occidentale du pays, celle d'Egbado, et ensuite celle d'Oyo. La tradition d'Egbado, plutôt rurale, est restée cantonnée à sa pratique familiale, c'est-à-dire le culte des reliques de famille, disparaissant en tant que mode d'initiation du « santo à l'africaine ». La tradition d'Oyo, principalement urbaine, a modifié les pratiques au point de faire disparaitre les plus anciennes ("santo à l'africaine »). Elle a surtout imposé le kariocha ou « santo couronné », qui est devenu par la suite l'initiation à proprement parler ${ }^{55}$. Si, dans 
le culte des reliques de famille, le lignage biologique se superpose au lignage rituel, à partir du moment où l'on introduit le processus initiatique, ce n'est plus le cas. Désormais, pour être inclus dans une famille de religion, il faudra suivre une initiation individuelle.

Dans la configuration actuelle de la pratique de la santería à Sagua, on peut penser que le «santo à l'africaine » a été un mode intermédiaire entre un culte familial et non-initiatique (les reliques de famille) et un autre, individuel et nécessitant une initiation (le santo à la cubaine), qui s'est imposé partout dans l'île. C'est cette même modalité du « santo créole » qui est devenue, au cours du $\mathrm{xx}^{\mathrm{e}}$ siècle, le paradigme de la santería cubaine. *

* Manuscrit reçu en janvier 2005, accepté pour publication en juin 2005.

\section{NOTES}

Je remercie Stefania Capone, Irina Georgieff et Pascale Jaunay pour leurs commentaires et suggestions.

1. La ville de Sagua la Grande se trouve sur le littoral nord de la région centrale de l'ile, à $260 \mathrm{~km}$ de la capitale du pays.

2. Depuis la période coloniale, l'ìle de Cuba est divisée en trois grandes régions : occidentale, centrale et orientale. La Havane et Matanzas se trouvent dans la région occidentale, Sagua la Grande dans la région centrale.

3. Cette enquête a été possible grâce à la collaboration de plusieurs santeros octogénaires et nonagénaires de la ville de Sagua (Testa 2004).

4. Ces institutions constituent l'un des sujets les plus étudiés dans l'ensemble de la culture afro-cubaine, une large profusion de textes dans le domaine étant la preuve de l'intérêt que suscite ce thème depuis plus de 150 ans.

5. La caja était une boîte destinée à garder l'argent que les membres apportaient pour le fonctionnement du cabildo.

6. À l'origine, le mot « créole » désignait toute personne de " race » blanche née dans les Antilles. Plus tard, son utilisation s'est élargie pour désigner aussi le descendant d'Africain né à Cuba. Dans le cadre de cette recherche, le terme " créole » est employé dans le second sens : il est également synonyme de Cubain. Pour une analyse détaillée du vocable « créole » voir Lavallé (1980).

7. Nicolas Angarica fut un disciple direct d'Obadimejí, premier obbá masculin (maître de cérémonie de la santería) selon Miguel Ramos (2003). Plusieurs orthographes sont possibles pour le nom Obadimeji ; on trouve également : Obadimelli, Obbadimelli, Obadimeyi, Obbadimeyi. Nous utilisons la dernière, c'est-à-dire Obbadimeyi.

8. On appelle santera et santero, la femme et l'homme qui ont été initiés dans la santería.

9. Les orichas sont les divinités adorées dans la santería.

10. Bien que les divinités soient d'origine yoruba, l’orthographe est totalement « cubanisée ».

11. La ville a eu une reconnaissance officielle le 8 décembre 1812 (Alcover y Beltrán 1905).

12. L'ordre national d'importance dans la production sucrière était Cárdenas (147 sucreries), Matanzas (128), Colón (126) et Sagua (119). Les trois premières appartiennent à la région occidentale, seule la ville de Sagua appartient à la région centrale.

13. Sagua la Grande et sa juridiction se trouvent dans cette province.

14. Un fundamento est un objet tel qu'une pierre ou un réceptacle avec différents éléments naturels et sur lequel est matérialisé un oricha ou une entité de mort. Le findamento doit être alimenté 
régulièrement avec du sang animal pour maintenir sa puissance. Les pièces rituelles utilisées dans la santería actuelle sont plus complexes que les reliques de famille : elles comportent plusieurs pierres et outils (herramientas) qui sont assemblés selon des règles plus ou moins fixes et qui diffèrent selon l'oricha auquel elles sont consacrées.

15. On dit qu'un fundamento " part » à la mort de son propriétaire. En effet, au moment du décès, la pièce est soit détruite, soit rendue à la nature. Dans certains cas, il arrive qu'au moment de la cérémonie funéraire (itutu) une ou plusieurs divinités demandent, par le biais de la divination, qu'une personne déterminée garde les autels individuels.

16. Dans la santería, toute naissance d'une pièce rituelle procède d'une autre pièce rituelle. On dira qu'elle « accouche » de nouvelles pièces.

17. Emilia est née en 1932, elle avait 68 ans au moment de l'entretien en 2000.

18. Le quartier de Pueblo Nuevo s'est construit sur des « terrains bas, insalubres et impraticables » (Alcover y Beltrán 1905, p. 544).

19. Raúl Villavicencio, directeur du Museo Histórico de Sagua, communication personnelle (21 décembre 1999).

20. À la mort de son dernier responsable, en 1990, la société San Francisco de Asís avait cessé ses activités. En 2002, elle a été réouverte sous l'ancien nom de cabildo Kunalumbo et le saint patron, saint François d'Assise, est maintenant syncrétisé avec Orula (dieu de la divination). Or ce syncrétisme ne correspond pas à l'univers congo mais à celui de la santería.

21. Pendant les dernières années du $\mathrm{XIX}^{\mathrm{e}}$ siècle, ainsi que pendant la période républicaine (19021958), la vie associative était très riche. Les Noirs ont créé de nombreuses sociétés pour leur progrès et leur divertissement, elles étaient regroupées dans le « Directoire général de sociétés noires de Cuba » (Directorio Central de Sociedades Negras de Cuba). À ce propos, voir Hevia Lanier (1996).

22. Certains auteurs considèrent que 1856 est l'année de l'introduction du spiritisme à Cuba. D'autres affïrment que la Guerre de Dix Ans (1868-1878) est la période de l'expansionnisme spirite, car les créoles insurgés (principalement dans la région orientale) avaient une tendance à assimiler cette pratique religieuse aux idées indépendantistes, en opposition au catholicisme qui était associé directement au pouvoir colonial espagnol (Argüelles et Hodge 1991).

23. Revue écrite et publiée à Sagua.

24. Pour la plupart des chercheurs cubains, le spiritisme arrive à Cuba via les États-Unis (Argüelles et Hodge 1991 ; Millet 1996; Hodge et Rodríguez 1997 ; Cordova et Barzaga 2000). Cependant, les publications spirites de Sagua la Grande ne font jamais référence aux liens entre les deux pays; au contraire, elles montrent les rapports qu'elles maintenaient avec des villes d'Amérique Latine et d'Europe.

25. Le babalao est le prêtre du culte d'Ifá, spécialiste de la divination, et se consacre au dieu Orula. Littéralement son nom veut dire « père (baba) du secret (awo) ".

26. Addechina est un des babalaos les plus renommés dans les prières (moyugbas) de ses confrères puisqu'il est considéré comme le père de l'Ifá cubain. Ifá étant l'oracle dont sont spécialistes les babalaos, c'est aussi un culte qui est fortement lié à la santería, tous les deux sont d'origine yoruba. Fernando Ortiz (1994, p. 146) reconnaît Addechina comme l'un des trois fondateurs du cabildo de Yemayá à Regla.

27. D’après Ramos (2003), la santeria de la fin du XIX et début du xx ${ }^{\mathrm{e}}$ siècle était entre les mains des femmes, notamment Latuán à La Havane et Obá Tero à Matanzas. Selon le même auteur, l'une et l'autre étaient très proches d'Addechina, voire " inséparables »; il est donc fort probable que l'une ou l'autre soit venue à Sagua pour ces initiations, mais la mémoire collective n'a retenu que le nom du babalao, ce qui est d'ailleurs en rapport avec la montée en force des hommes dans la religion pendant le $\mathrm{xx}^{\mathrm{e}}$ siècle.

28. Elle fut aussi la marraine du peintre surréaliste Wifredo Lam, né à Sagua la Grande en 1902 dans le quartier de Cocosolo.

29. Les termes « filleule " (ahijada) pour la femme et « filleul » (ahijado) pour l'homme désignent le lien de parenté (rituelle) unissant une personne à celle qui l'initie dans la santería. Cette dernière sera 
désormais sa "marraine " (madrina) si c'est une femme ou son "parrain " (padrino) si c'est un homme. Ces mêmes mots sont utilisés pour les liens de parenté créés à la suite du baptême catholique, que nous écrivons sans guillemets pour les différencier de ceux utilisés dans la santería.

30. Iyaworage est le nom de l'année qui suit la date de l'initiation à la santería, iyawó est le nouvel initié qui est en période d'iyaworage. Des obligations et des interdictions sont imposées à l'ijawó ; leur non-respect entraînerait le châtiment de la part des orichas. Pour les mêmes termes, on trouve d'autres orthographes : yaworage, ijaguorage ; yawó, yaguó.

31. Santo et oricha sont deux termes synonymes dans la santería, les pratiquants utilisant davantage celui de santo.

32. L'expression "faire une œuvre" (hacer una obra, hacer um trabajo) indique, dans ce cas, " réaliser une offrande, un nettoyage rituel ou une cérémonie précise auprès d'une divinité ".

33. La santería est régie par un principe de séniorité : l'ancienneté dans une fonction concède prestige et pouvoir, par conséquent (entre pratiquants qui ont le même degré d'initiation) une prééminence du plus ancien sur les autres.

34. La capitale Oyo fut rasée en 1837, à la suite de quoi la population se dispersa et le royaume d'Oyo tomba dans l'anarchie (Tamisier 1998).

35. La tradition d'Egbado à Matanzas est appellée "santo parado " ou " santo de dotación " (Ramos 2003).

36. Une situation semblable est arrivée à Obadimeji, fils de lucumí, originaire de Sabanillas. À son arrivée à La Havane, il a été contraint de s'initier une nouvelle fois car la première initiation n'était pas reconnue dans la capitale (Ramos 2003).

37. Dans les liens de parenté rituelle qui s'établissent lors de l'initiation dans la santería, l'oy'ugbona est la personne qui, entre autres, seconde la « marraine » et la remplace en cas de décès. De ce fait, elle est également appelée " deuxième marraine ». On trouve aussi yugbona, oyubona, oyyugbona.

38. Omó Changó signifie " initié au culte de Changó »: omó vient du yoruba qui veut dire " fils ».

39. Pour les termes « marraine » et " parrain », voir la note 29.

40. L'obbá-oriaté est le maître de cérémonie dans la santería ; il dirige tous les rituels lors d'une initiation. C'est aussi un spécialiste de la divination par les cauris.

41. D’après Jesús López Zúñiga, la chanson disait " Iyamuana Mundele, Muana Mundele Kitamamba », ce qui signifie " un homme blanc vient saluer ».

42. Jesús López Zúñiga est né en 1917, il avait 83 ans au moment de l'entretien en 2000.

43. Pedro Pablo Dreke Alfonso est né en 1922, il avait 78 ans au moment de l'entretien en 2000.

44. Les quatre autres étaient José Manuel Gibet, Pepe, omó Changó ; Concepción Gibet, Conchita, omó Ochún ; Valeriana Pequeño, omó Yemayá, et Elvira Mestre, omó Ochún.

45. Tomás Romero (Ewinleti), omó Obatalá, est reconnu par Valdés Garriz (1997, p. 135) comme l'un des premiers oriatés de Cuba et aussi comme un des grands connaisseurs du dilogín. Il s'agit d'un système divinatoire utilisé dans la santería et se servant de cauris pour avoir accès à l'oracle.

46. Obbasa-oriaté est le féminin d'obbá-oriaté (maître de cérémonie de la santería). Elles ont existé de la fin du $\mathrm{xIx}^{\mathrm{e}}$ siècle au début du $\mathrm{Xx}^{\mathrm{e}}$.

47. Rómulo Lachatañeré fut le premier à contester l'utilisation du vocable " sorcellerie " (brujería) pour se référer aux pratiques religieuses des descendants d'Africains. Ce vocable était en vogue au début du $\mathrm{xx}^{\mathrm{e}}$ siècle ; les premiers écrits de Fernando Ortiz en témoignent amplement. Lachatañeré proposait à la place le terme de "santería ", ce qui était sans doute une légitimation des cultes considérable dans la société cubaine de l'époque. À ce propos voir Erwan Dianteill (1995) qui fait une analyse approfondie de la naissance de l'étude scientifique des religions afro-cubaines.

48. Pour certains santeros havanais, le santo est l'ensemble des cauris (parce que c'est à travers eux que le santo parle) et non les pierres, ce qui confirme l'importance de ce changement.

49. La première « filleule » de Deogracia Herrera raconte que sa marraine disait que, lors de son initiation avec Gregorio Torregrosa, son oricha n'a pas accepté d'autre nom rituel pour sa fille que celui de sa première « marraine », Elena Mestre. Toutes les deux étaient des filles de Changó et avaient pour nom rituel Arokumatindé. 
50. La présentation au tambour consacré ( $A \tilde{n} a ̄$ ) du nouvel initié (iyawó) est une cérémonie très importante parce que c'est à ce moment-là qu'Olofin, dieu suprême, reconnaît la personne en tant que santero.

51. La société avait son siège social dans le quartier havanais de Los Sitios, à l'adresse du domicile personnel de son président.

52. Reglamento de la Sociedad Culto Lucumi San Pedro Crisólogo, 1937 (dossier 313, " negroscabildos », Fond Fernando Ortiz, Instituto de Literatura y Lingüística de La Habana).

53. Omó Añá, traduit par « fils du tambour », est le nom donné au musicien qui est initié au culte d'Añá. On dit aussi batalero ou tamborero. Il est le seul autorisé à jouer des tambours consacrés.

54. La société Santa Bárbara de Villa Alegre est, depuis ses origines, un lieu d'initiation à la santería. Elle a connu les mêmes avatars que les autres, avec des initiations à l'africaine d'abord et créoles ensuite. Néanmoins sa filiation n'est pas havanaise, mais de Matanzas, de la grande famille religieuse de Ferminita Gomez (Ocha Bí), héritière de Ma' Monserrate González (Obá Tero), importante obbasa-oriaté lucumí du Xix ${ }^{e}$ siècle et décédée en 1907.

55. La pratique de la santería est un thème bien étudié par les chercheurs cubains et étrangers. À Cuba, la bibliographie sur le sujet est si prolixe qu'un catalogue réalisé par Fernandez Robaina lui a été consacré en 1985. Depuis, elle ne cesse de croître, aussi bien dans l'île qu'ailleurs, notamment aux États-Unis. En France, deux ouvrages ont été publiés, l'un dans une optique anthropologique (Argyriadis 1999), l'autre sociologique (Dianteill 2000).

\section{BIBLIOGRAPHIE}

Alcover y Beltrán Miguel Antonio

1905 Historia de la villa de Sagua la Grande y su jurisdicción, Imprentas unidas La Historia y el Correo Español, Sagua la Grande.

ANGarica Nicolás

1990 "El lucumí al alcance de todos », in Lázara Menéndez, éd., Estudios afrocubanos, vol. 4, pp. 3-128, Universidad de La Habana, La Havane.

Argüelles Mederos Aníbal et Ileana Hodge Limonta

1991 Los llamados cultos sincréticos y el espiritismo, Editorial Academia, La Havane.

\section{ArgYriadis Kali}

1999 La religión à La Havane. Actualité des représentations et des pratiques cultuelles havanaises, Éditions des archives contemporaines, Paris.

\section{Córdova Martínez Carlos et Oscar Barzaga Sablon}

2000 El espiritismo de cordón, Fundación Fernando Ortiz, La Havane.

\section{Deschamps Chapeaux Pedro}

1969 "Marcas tribales de esclavos en Cuba », Etnología y Folklore, 8, pp. 63-78, La Havane.

1971 El negro en la economía habanera del siglo xIx, UNEAC, La Havane.

DiANTEILl Erwan

1995 Le savant et le santero. Naissance de l'étude scientifique des religions afrocubaines (1906-1954), Éditions L'Harmattan, Paris.

2000 Des dieux et des signes. Initiation, écriture et divination dans les religions afro-cubaines, Éditions de l'EHESS, Paris. 


\section{FERnÁNDEZ RobaINA Tomás}

1985 Bibliografia de temas afrocubanos, Biblioteca Nacional José Martí, La Havane.

\section{Hevia Lanier Oilda}

1996 El Directorio Central de las Sociedades Negras de Cuba (1886-1894), Editorial de Ciencias Sociales, La Havane.

2002 "Los cabildos de nación en la provincia de Matanzas », conférence Ve Taller Internacional «Problemas teóricos y prácticos de la historia regional y local », 22-24 abril, Instituto de Historia, La Havane.

Hodge Limonta Ileana et Minerva Rodríguez Delgado

1997 El espiritismo en Cuba, Editorial Academia, La Havane.

LACHATAÑeré Rómulo

2001 El sistema religioso de los afrocubanos, Editorial de Ciencias Sociales, La Havane.

LAVALlé Bernard

1980 "De "l'esprit colon" à la revendication créole ", in J. Pérez et al., Esprit créole et conscience nationale, Éditions du CNRS, Paris.

Millet José

1996 El espiritismo, variantes cubanas, Editorial Oriente, Santiago de Cuba.

Montejo ARrechea Carmen

1993 Sociedades de instrucción y recreo de pardos y morenos que existieron en Cuba colonial, 1878-1898, Instituto Veracruzano de Cultura, Veracruz, Mexique.

Moreno Fraginals Manuel

1978 El ingenio, Editorial de Ciencias Sociales, La Havane.

OrTIz Fernando

1906 Los negros brujos, Editorial de Ciencias Sociales, La Havane.

1993 «Los cabildos afrocubanos », in Etnia y sociedad, chap. 7, pp. 54-63, Editorial de Ciencias Sociales, La Havane.

1994 Los tambores batá de los yorubas, Publicigraf, La Havane, coll. « Raíces ».

Pichardo Esteban

1985 Diccionario provincial casi razonado de voces y frases cubanas, Editorial de Ciencias Sociales, La Havane [1849].

Ramos Miguel W.

2003 "La división de La Habana : territorial conflict and cultural hegemony in the followers of Oyo Lukumí religion, 1850s-1920s », Cuban Studies, 34, pp. 3870, University of Pittsburgh Press.

Rebello Carlos

1860 Estados relativos a la producción azucarera de la Isla de Cuba, [s.n.], La Havane.

ScotT Rebecca

2001 La emancipación de los esclavos en Cuba, Editoral Caminos, La Havane. 
TAMISIER Jean-Christophe, éd.

1998 Dictionnaire des peuples, Larousse, Paris.

Testa Silvina

2004 Como una memoria que dura. Cabildos, sociedades y religiones afrocubanas de Sagua la Grande, Ediciones La Memoria, La Havane, coll. «Coloquios y testimonios ».

VALDES GaRRIZ Yrmino

1997 Dilogún, Ediciones Unión, La Havane.

Venegas Delgado Hernán

1989 Aproximaciones y diferencias del desarrollo económico-social de Las Villas, thèse de doctorat en histoire, Universidad Central de Las Villas, Cuba. 